\title{
Reconstructions and phase transitions of the Ge(001) surface
}

\author{
H J W Zandvliet $†$, D Terpstra and A van Silfhout \\ Faculty of Applied Physics, University of Twente, PO Box 217, 7500 AE Enschede, \\ The Netherlands
}

Received 7 March 1990, in final form 24 July 1990

\begin{abstract}
We have calculated the energy differences between the four members of the $2 \times 1$ family $(b(2 \times 1), c(4 \times 2), p(2 \times 2)$ and $p(4 \times 1))$ of the $G(001)$ surface by taking only electrostatic interactions between the dimers into account. We have found that $p(2 \times 2)$ is the lowest-energy reconstruction at zero temperature.

The phase transitions from ordered $\mathrm{p}(2 \times 2)$ to disordered $(2 \times 1)$ and ordered $\mathrm{c}(4 \times 2)$ to disordered $(2 \times 1)$ are discussed using our energy calculations, the mean-field approximation, the Ising model and Onsager's exact solution. A second-order phase transition is found at about $200-250 \mathrm{~K}$ from an ordered $\mathrm{p}(2 \times 2)(\mathrm{c}(4 \times 2))$ dimer reconstruction to a disordered $(2 \times 1)$ dimer reconstruction assuming a charge transfer of $0.08 \mathrm{e}$ between the up atom and the down atom of the asymmetric dimer.
\end{abstract}

\section{Introduction}

There has been considerable theoretical and experimental interest in the electronic and geometric structure of group IV semiconductor surfaces. The germanium and silicon (001) surfaces are the simplest and yet still controversial systems, which have been investigated for about 30 years since Schlier and Farnsworth [1] first provided evidence for top-layer atom pairing, leading to a $(2 \times 1)$ reconstruction. Recently the $\mathrm{Ge}(001)$ surface has been investigated using scanning tunnelling microscopy (STM) [2]. An asymmetric dimer reconstruction is observed that does not require vacancy-type defects for stabilization at room temperature. Regions of local $(2 \times 1), c(4 \times 2)$ and $p(2 \times 2)$ symmetry are found and the atomic positions in these regions are modelled using different arrangements of asymmetric buckled dimers. The dimers are thought to be asymmetric in the sense that the dimer bond axis is not parallel to the surface plane: one atom moves away from the surface, while the other moves in [3-5]. Lambert et al [6], using He diffraction, report $\mathrm{c}(4 \times 2)$ and $\mathrm{p}(2 \times 2)$ symmetries at $T<150 \mathrm{~K}$ in agreement with the work of Kevan [7], who observed a low-energy electron diffraction (LEED) diffraction peak indicative of either $\mathrm{p}(2 \times 2)$ or $\mathrm{c}(4 \times 2)$ at $220 \mathrm{~K}$. However, this disagrees with the results of Culbertson et al [8], who report only $\mathrm{c}(4 \times 2)$ diffraction patterns at low temperature. Rich et al [9] experimentally estimated that the charge transfer between the dimer atoms of $\mathrm{Ge}(001)$ is not more than 0.1 e. For the closely 


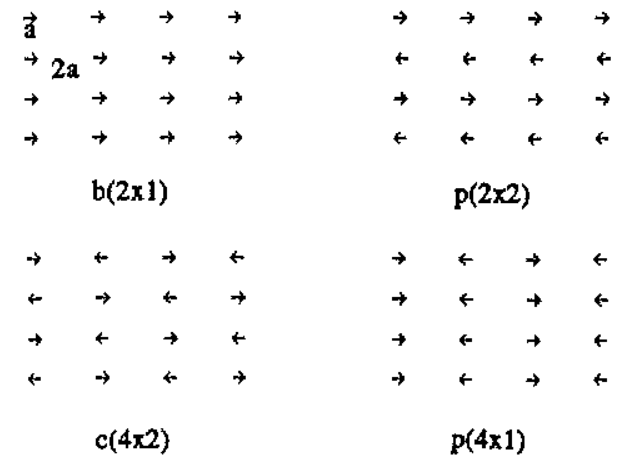

Figure 1. Reconstruction geometries of the Ge(001) surface for the $(2 \times 1)$ fanily. The tilting of the dimers is $14^{\circ}[4]$ : their length is $2.45 \not$ [4, 13]. The character of the dimers (tilt angle and bond length $[3,4,13\})$ is the same for all asymmetric dimer reconstructions. The surface lattice constant $u$ is $4.00 \bar{A}$.

related $\mathrm{Si}(001)$ surface the charge transfer between the down atom and the up atom has been estimated by Chadi [10] (0.36e), Rich et al [11] (less than $0.1 \mathrm{e}$ ) and very recently by Ong and Chan [12] $(0.11 \mathrm{e}-0.16 \mathrm{e})$.

$A b$ initio calculations of the total energies of the $\mathrm{Ge}(001)$ reconstructions have been performed $[3,4]$. showing that $\mathrm{p}(2 \times 2)$ and $\mathrm{c}(4 \times 2)$ are the lowest-energy reconstructions being nearly degenerate in energy. An earlier calculation [5] using an effective spin Hamiltonian and tight-binding calculations essentially yields the same result for the $\mathrm{Si}(001)$ surface. The analogy to an Ising spin system is obvious; the ordered $(2 \times 1)$ structure corresponds to the ferromagnetic phase, while the $\mathrm{c}(4 \times 2)$ structure corresponds to the antiferromagnetic phase (figure 1 ).

It is the aim of the present paper to show that the $\mathrm{p}(2 \times 2)$ reconstruction is evidently lower in energy than the $c(4 \times 2)$ reconstruction at zero temperature. We shall show that the energy differences between the four members of the $(2 \times 1)$ family of the $\mathrm{Ge}(001)$ surface can be calculated by taking only electrostatic interactions between the asymmetric dimers into account. Furthermore the transition temperatures for the ordered $\mathrm{p}(2 \times 2)$ to the disordered $(2 \times 1)$ reconstruction and for the ordered $\mathrm{c}(4 \times 2)$ to the disordered $(2 \times 1)$ reconstruction are calculated. The phase transition temperatures are in very good agreement with experimental data [7] assuming a charge transfer of about $0.08 \mathrm{e}$ between the down atom and the up atom of the asymmetric dimer.

\section{Calculational procedure and results}

We compute the electrostatic energy for four members of the $(2 \times 1)$ family of buckled dimer reconstructions (figure 1). The asymmetric dimer model is used [2-4]; pairs of atoms at the surface relax by dimerization into an asymmetric configuration. Associated with the asymmetric dimer are two characteristic surface states: one filled $\left(D_{\mathrm{up}}\right)$ and one empty $\left(D_{\text {down }}\right)$. This can also be seen as a net charge transfer [9] from the lower to the higher part of the dimer and this corresponds to a dipole of length $2.45 \AA$ and a tilt angle of $14^{\circ}[3,4,13,14]$. Theoretical calculations [3,4] indicate that the dimer tilt angle and that the dimer bond length are approximately the same for all different members of the $(2 \times 1)$ family; therefore the energy differences between the reconstructions are determined by the ordering of the dimers only. Our basic building block, the asymmetric dimer or dipole, contains two Ge surface atoms and has two possible orientations [14].

The energy difference $\delta U$ between one configuration and another can, for symmetry reasons, be found by switching one and only one dipole of the surface cell, at a time. 
Table 1. Energy differences of the $(2 \times 1)$ family symmetry configurations of the Ge(001) surface. $(\Gamma<0.1$ [9].) Data taken from $[3,4,14,15]$.

\begin{tabular}{lrccc}
\hline & \multicolumn{4}{c}{ Energy difference $(\mathrm{eV} /$ dimer) } \\
\cline { 2 - 5 } Reconstruction & \multicolumn{1}{c}{$[15]$} & \multicolumn{1}{c}{$[3]$} & \multicolumn{1}{c}{$[4]$} & This work \\
\hline $\mathrm{b}(2 \times 1)$ & 0.000 & 0.00 & 0.000 & \multicolumn{1}{c}{0.00} \\
$\mathrm{c}(4 \times 2)$ & -0.066 & -0.05 & -0.066 & $-1.95 \Gamma^{2}$ \\
$\mathrm{p}(2 \times 2)$ & -0.070 & & -0.069 & $-2.25 \Gamma^{2}$ \\
$\mathrm{p}(4 \times 1)$ & 0.036 & & 0.035 & $3.23 \Gamma^{2}$ \\
\hline
\end{tabular}

The non-switching dipole will be denoted $p_{0}$ and is located at the origin. The electric field $E_{i}(\mathbf{0})$ at the origin caused by the other dipoles, located at $\boldsymbol{r}_{1}$, is treated as follows. If $\left|r_{i}\right|$ exceeds $R$, which in our calculations is about $240 \AA$, the dipoles are point like; otherwise the dipoles (also $p_{i j}$ ) are replaced by two discrete charges, $q$ and $-q$. Since the $(2 \times 1)$ reconstruction is used as the energy reference, the following expressions will yield the energy differences $\delta U(n \times m)$, where $(n \times m)$ indicates the reconstruction:

$$
\begin{aligned}
& \delta U=U[(n \times m)]-U[(2 \times 1)] \\
& U=-\sum_{\left|r_{i}\right|>R} p_{0} \cdot E_{i}(\mathbf{0})-q \sum_{\left|r_{i}\right|<R} V_{i}\left(\frac{-L}{2}\right)+q \sum_{\left|r_{i}\right|<R} V_{i}\left(\frac{L}{2}\right) \\
& p=q L=\Gamma e L \\
& E_{i}(0)=\left(1 / 4 \pi \varepsilon_{0}\right)\left\{\left[\left(3 r_{i} \cdot p_{i}\right) /\left|r_{i}\right|^{5}\right] r_{i}-p_{i} /\left|r_{i}\right|^{3}\right\} \\
& V_{i}(x)=q_{i} / 4 \pi \varepsilon_{0}\left|r_{i}-x\right| \quad x=-L / 2 \text { or } x=L / 2
\end{aligned}
$$

where $L$ is the dimer length, $\Gamma$ is the charge transfer from the down atom to the up atom of the asymmetric dimer and $\varepsilon_{0}$ is the permittivity of free space $\left(8.854 \times 10^{-12} \mathrm{~F} \mathrm{~m}^{-1}\right)$. $E_{i}(0)$ is the electric field caused at the origin by a dipole $p_{t}$ at $r_{i}$ and $V_{1}$ the corresponding electrostatic potential. The summation runs over all surface lattice sites with the exception of the origin. Since the ordering of the energy differences has to be the ordering of the total energy, the energetically favourable reconstruction at $T=0 \mathrm{~K}$ can be found.

Table 1 shows the energy differences $\delta U(n \times m)$ in electron volts per dimer. The physical reason why the $\mathrm{p}(2 \times 2)$ reconstruction is lower in energy than the $\mathrm{c}(4 \times 2)$ reconstruction is because an in-phase ordering of adjacent rows of dipoles is energetically preferred to out-of-phase ordering. So the ordering of the energies of the different reconstructions relative to each other can be understood on the basis of electrostatic arguments only. The alternation of dimers along a row makes the $p(2 \times 2)$ and $c(4 \times 2)$ symmetry reconstructions lower in energy than the $b(2 \times 1)$ and $\mathrm{p}(4 \times 1)$ symmetry reconstructions.

Assuming a charge transfer of about $\Gamma=0.15$ for the $\mathrm{Ge}(001)$ surface we get essentially the same results as the ab initio calculations of Needels et al $[3,4]$ and Payne et al [15]. (Rich et al [9] estimated a lower charge transfer: $\Gamma<0.1$.) It is interesting to note that the results of the closely related $\mathrm{Si}(001)$ surface [5] are essentially the same $(\mathrm{b}(2 \times 1)$, 


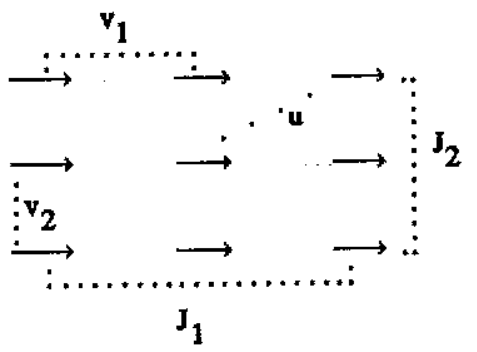

Figure 2. Effective couplings between adjacent dimers.

$0 \mathrm{eV} /$ dimer; $\mathrm{c}(4 \times 2),-0.031 \mathrm{eV} /$ dimer; $\mathrm{p}(2 \times 2),-0.036 \mathrm{eV} /$ dimer; $\mathrm{p}(4 \times 1)$, $0.036 \mathrm{eV} /$ dimer $)$.

\section{Phase transitions}

With increasing temperature $T$ the phase transitions of the $\mathrm{Ge}(001)$ and $\mathrm{Si}(001)$ surfaces occur from ordered reconstructed phases to the disordered $(2 \times 1)$ reconstruction [6$8,16-18]$. In this paper the phase transitions from ordered $c(4 \times 2)$ to disordered $(2 \times 1)$ and from ordered $\mathrm{p}(2 \times 2)$ to disordered $(2 \times 1)$ are considered. Our basic approximations are summarized as follows.

(i) Different reconstructions result from different arrangements of asymmetric dimers which are the building blocks of the surface in this model.

(ii) The electrostatic differences for the four members of the $(2 \times 1)$ family are calculated using our dipole model [14].

In the case when we map the different reconstructions of the $\mathrm{Ge}(001)$ surface onto the spin Ising Hamiltonian, we also have the following approximation.

(iii) The possible orientations of the asymmetric dimer are represented by the two possible states of a spin $[5,16]$. For the Ising spin $s_{x, y}= \pm 1$ corresponding to these orientations (here $x, y$ denote the lattice sites in the $x$ and $y$ directions) the dimer lattice converts to the rectangular Ising lattice.

Ihm etal [5] showed that interactions of at least three nearest-neighbour coordination spheres must be included in the effective spin Hamiltonian for Si(001). Thus, the model Hamiltonian describing the reconstruction of $\mathrm{Ge}(001)[16]$ and $\mathrm{Si}(001)$ can be represented as (figure 2 )

$$
\begin{aligned}
H=-\frac{1}{2} \sum_{x, y} s_{x, y} & {\left[v_{1}\left(s_{x-1, y}+s_{x+1, y}\right)+v_{2}\left(s_{x, y-1}+s_{x, y+1}\right)\right.} \\
& +u\left(s_{x-1, y-1}+s_{x-1, y+1}+s_{x+1, y-1}+s_{x+1, y+1}\right) \\
& \left.+J_{1}\left(s_{x-2, y}+s_{x+2, y}\right)+J_{2}\left(s_{x, y-2}+s_{x, y+2}\right)\right]
\end{aligned}
$$

where the summation is taken over all lattice sites. In comparison with [5], two additional terms with $J_{1}$ and $J_{2}$ are taken into account. They correspond to interactions between second-nearest neighbours along the $x$ and $y$ directions. The terms involving $s_{x, y} s_{x, y+1} s_{x+1, y} s_{x+1, y+1}$ interactions contribute equally to the total energies for all four symmetries and are initially set equal to zero (the same holds in principle for $J_{1}+J_{2}$ ).

To obtain the relations between the interaction constants, the mean energies $E_{i}=$ 
Table 2. Total energies for the $(2 \times 1)$ family of the $\mathrm{Ge}(001)$ surface (per spin in the Ising model and per dimer in the dipole model). The disordered non-buckled $(2 \times 1)$ reconstruction is taken as the zero of energy.

\begin{tabular}{llllc}
\hline \multicolumn{2}{c}{ Phase notation } & & \multicolumn{2}{c}{ Energy } \\
\cline { 5 - 6 } \cline { 5 - 6 } \cline { 5 - 6 } Magnetic & Structural & $\begin{array}{l}\text { Ising model } \\
\text { (per spin) }\end{array}$ & $\begin{array}{l}\text { Dipole model } \\
\text { (per dimer) }\end{array}$ \\
\hline Ferromagnetic & $\mathrm{b}(2 \times 1)$ & $-v_{1}-v_{2}-2 u-\left(J_{1}+J_{2}\right)$ & $0.645 \Gamma^{2}$ \\
Antiferromagnetic & $\mathrm{c}(4 \times 2)$ & $v_{1}+v_{2}-2 u-\left(J_{1}+J_{2}\right)$ & $-1.309 \Gamma^{2}$ \\
Layered antiferromagnetic a & $\mathrm{p}(2 \times 2)$ & $-v_{1}+v_{2}+2 u-\left(J_{1}+J_{2}\right)$ & $-1.610 \Gamma^{2}$ \\
Layered antiferromagnetic b & $\mathrm{p}(4 \times 1)$ & $v_{1}-v_{2}+2 u-\left(J_{1}+J_{2}\right)$ & $3.868 \Gamma^{2}$ \\
\hline
\end{tabular}

$\left\langle H_{i}\right\rangle$ of various ordered phases at $T=0 \mathrm{~K}$ should be compared. The energies of four ordered structures are presented in table 2 . The $T=0 \mathrm{~K}$ values of $v_{1}, v_{2}$ and $u$ can be derived from the energy differences of the four configurations in the dipole model $\left(v_{i}=\right.$ $882 \Gamma^{2} \mathrm{meV}, v_{2}=-1857 \Gamma^{2} \mathrm{meV}$ and $u=366 \Gamma^{2} \mathrm{meV}$ ). As can be seen by the relative magnitude of $v_{1}, v_{2}$ and $u$, the strongest coupling between dimers is along the rows. (The $\operatorname{sum} J_{1}+J_{2}=-402 \Gamma^{2} \mathrm{meV}$ does not depend on the energy differences between the four members of the $(2 \times 1)$ family; see table 2 .)

Theoretical calculations $[4,5,16,17]$ predict that a second-order phase transition occurs between layered antiferromagnetic $(\mathrm{p}(2 \times 2)$ [5]) or antiferromagnetic $(\mathrm{c}(4 \times 2)$ $[16,17])$ to a paramagnetic (disordered) phase at roughly $380 \pm 100 \mathrm{~K}$ for $\mathrm{Ge}(001)[4]$ and $250 \mathrm{~K}$ for $\mathrm{Si}(001)[5,17]$. This conclusion agrees with the STM work of Kubby et al [2], who showed that domains of $b(2 \times 1)$ and $p(2 \times 2)$ coexist with $c(4 \times 2)$ at room temperature for the $\mathrm{Ge}(001)$ surface. Zubkus and Tornau [16] considered the phase transition from the disordered $(2 \times 1)$ phase to the ordered $c(4 \times 2)$ and $p(2 \times 2)$ phases using the mean-field approximation. The transition temper atures $T_{\mathrm{lc}}(\mathrm{p}(2 \times 2)$ to disordered $(2 \times 1))$ and $T_{2 c}(\mathrm{c}(4 \times 2)$ to disordered $(2 \times 1))$ using the equations derived by Zubkus and Tornau [16] and our interaction constants are given by

$$
\begin{aligned}
& k T_{\mathrm{lc}}=2\left(v_{1}-v_{2}-2 u+J_{1}+J_{2}\right)=3.2(e \Gamma)^{2} \\
& \left(T_{1 \mathrm{c}}=237 \mathrm{~K} \quad \Gamma=0.08\right) \\
& k T_{2 \mathrm{c}}=2\left(-v_{1}-v_{2}+2 u+J_{1}+J_{2}\right)=2.6(e \Gamma)^{2} \\
& \left(T_{2 \mathrm{c}}=192 \mathrm{~K} \quad \Gamma=0.08\right) .
\end{aligned}
$$

As an example we have substituted a $\Gamma$-value of 0.08 [9] into equations (7) and (8), resulting in transition temperatures of $200-250 \mathrm{~K}$ (in all the phase transition temperatures given below we have substituted this $\Gamma$-value of 0.08 ). When we include only nearest-neighbour interactions $\left(v_{1}, v_{2}\right)$ the exact solution of Onsager can be used giving a phase transition temperature of about $221 \mathrm{~K}$ (see appendix 1).

We shall show below that it is not necessary to map the energies of the four different asymmetric dimer reconstructions onto the Ising spin Hamiltonian. The simplest approximation is to calculate the energy to flip one dimer in the odrered low-temperature reconstruction, to keep all the other dimers fixed in their ordered reconstruction [7] and to compare this energy with $k T$. The energy to flip one dimer in the dipole model of the ordered $\mathrm{p}(2 \times 2)(\mathrm{c}(4 \times 2))$ results in roughly the same phase transition temperature: $T_{1 \mathrm{c}}=281 \mathrm{~K}\left(T_{2 \mathrm{c}}=239 \mathrm{~K}\right)$ compared with the results of the Ising spin phase transition 
temperatures. The mean-field approximation can also be used directly in the ferroelectric-antiferroelectric case, resulting in the following phase transition temperatures: $T_{1 \mathrm{c}}=239 \mathrm{~K}$ and $T_{2 \mathrm{c}}=195 \mathrm{~K}$ (see appendix 2 ).

Summarizing we can say that all models (mean-field approximation, Onsager's exact solution and a simple dimer flip) give approximately the same phase transition temperatures. Assuming a charge transfer of about $0.08 \mathrm{e}$ [9] the predicted phase transition temperatures are in good agreement with the LEED measurements of Kevan [7], which indicate that a second-order phase transition from ordered $c(4 \times 2)($ and $p(2 \times 2))$ to disordered $(2 \times 1)$ takes place at about $220-260 \mathrm{~K}$ for the $\mathrm{Ge}(001)$ surface.

\section{Conclusions}

We have calculated the energy differences of four different asymmetric dimer reconstructions of the $\mathrm{Ge}(001)$ surface at zero temperature. We have shown that by computing the electrostatic energy of the dimers of one layer we get essentially the same results as emerged from the $a b$ initio density-functional calculations of Needels et al $[3,4]$. Payne et al [15] and from the total-energy renormalization group approach of $\mathrm{Ihm}$ et al [5] for the Si(001) surface. However, these workers state that the difference between the $\mathrm{p}(2 \times 2)$ and $\mathrm{c}(4 \times 2)$ asymmetric dimer reconstructions is within the uncertainties in their calculations, whereas we believe, on the basis of our calculations, that the $p(2 \times 2)$ is essentially lower in energy than the $c(4 \times 2)$ reconstruction. From our energy calculations we derive coupling parameters for a simple two-dimensional Ising spin model for the asymmetric dimers and predict a phase transition temperature of $200-250 \mathrm{~K}$ for ordered $\mathrm{c}(4 \times 2)$ or $\mathrm{p}(2 \times 2)$ to a disordered (paramagnetic) $(2 \times 1)$ phase (assuming a charge transfer of $0.08 \mathrm{e}$ [9]), in agreement with experimental data and other theoretical calculations. Within the dipole model, use of the mean-field approximation results in approximately the same phase transition temperatures.

\section{Appendix 1}

The exact Onsager solution in two dimensions (only the nearest-neighbour interaction) is given by (we have assumed a $\Gamma$-value of 0.08 ) [19]

$$
\begin{aligned}
& 1=\sinh \left(2\left|v_{1}\right| / k T_{\mathrm{c}}\right) \sinh \left(2\left|v_{2}\right| / k T_{\mathfrak{c}}\right) \\
& 1=\sinh \left(T_{\mathrm{c}}=221 \mathrm{~K}, 130.9 / T_{\mathrm{c}}\right) \sinh \left(275.6 / T_{\mathrm{c}}\right)
\end{aligned}
$$

\section{Appendix 2}

The following equation can be derived:

$$
U=-\frac{1}{2} \sum_{i, 1} p_{i} \cdot E_{j}
$$

with

$$
\begin{aligned}
& E_{j}=\left(1 / 4 \pi \varepsilon_{0}\right)\left[\left(3 \boldsymbol{r}_{i j} \cdot \boldsymbol{p}_{j}\right) /\left|\boldsymbol{r}_{i j}\right|^{5}\right] \boldsymbol{r}_{i j}-\boldsymbol{p}_{j} /\left|\boldsymbol{r}_{i j}\right|^{3} \\
& \langle\boldsymbol{p}\rangle=\tanh \left(\sum_{j} \frac{p^{2}}{2 \pi \varepsilon_{0}\left|\boldsymbol{r}_{j}\right|^{3}} \frac{3 \cos ^{2} \alpha_{j}-1}{k T}\langle p\rangle\right)
\end{aligned}
$$


and $p= \pm 1$ give

$$
k T_{\mathrm{c}}=\sum_{j} \frac{p^{2}}{2 \pi \varepsilon_{0}\left|\boldsymbol{r}_{j}\right|^{3}}\left(3 \cos ^{2} \alpha_{j}-1\right) .
$$

The following antiferroelectric $(\mathrm{p}(2 \times 2)$ or $\mathrm{c}(4 \times 2))$ to paraelectric $(2 \times 1)$ phase transition temperatures are obtained: $T_{1 \mathrm{c}}=239 \mathrm{~K}$ and $T_{2 \mathrm{c}}=195 \mathrm{~K}$.

\section{References}

[1] Schlier RE and Farnsworth H E 1959 J. Chem. Phys. 30917

[2] Kubby J A, Griffith J E, Becker R S and Vickers J S 1987 Phys. Rev. B 366079

[3] Needels M, Payne M C and Joannopoulos J D 1987 Phys, Rev. Lett. 581765

[4] Needels M, Payne M C and Joannopoulos J D 1988 Phys. Rev. B 385543

[5] Ihm J. Lee D H. Joannopoulos J D and Xiong J J 1983 Phys. Rev. Lett. 511872

[6] Lambert W R, Trevor P L, Cardillo M J, Sakai A and Hamann D R 1987 Phys. Rev. B 358055

[7] Kevan S D 1985 Phys. Rev. B 322344

[8] Culbertson R J. Kuk Y and Feldman L C 1986 Surf. Sci. 167127

[9] Rich R D, Miller T and Chiang T-C 1988 Phys. Rev. Lett. 60357

[10] Chadi D J 1979 Phys. Rev. Lett. 4343

[11] Rich D H, Miller T and Chiang T-C 1988 Phys. Rev. B 373124

[12] Ong C K and Chan B C 1989 J. Phys.: Condens. Matter 13931

[13] Grey F, Johnson R L. Skov Pedersen J, Feidenhans"1 R and Nielsen M 1987 Proc. 2nd Int. Conf. on the Surface of Surfaces (Amsterdam) (Springer Series in Surface Sciences) vol 11, eds J F van der Veen and $M A$ van Hove (Berlin: Springer) pp 292-7

[14] Zandvliet H J W, Poppe G P M. Wijers C M J and van Silfhout A. 1989 Solid State Commun. 7163

[15] Payne M C, Roberts N, Needs R J, Needels M and Joannopoulos J D 1989 Surf. Sci. 211-2 1

[16] Zubkus V E and Tornau E E 1989 Surf. Sci. 21623

[17] Saxena A, Gawlinski E T and Gunton J D 1985 Surf. Sci. 160618

[18] Tabata T, Aruga T and Murata Y 1987 Surf. Sci. 179 L63

[19] McCoy B M and Wu T T 1973 The Two-dimensional Ising model (Cambridge, MA: Harvard University Press) p 80 